\title{
Image Gradient Based Level Set Methods in 2D and 3D
}

\author{
Xianghua Xie, Si Yong Yeo, Majid Mirmehdi, Igor Sazonov, and Perumal \\ Nithiarasu
}

\begin{abstract}
This chapter presents an image gradient based approach to perform 2D and $3 \mathrm{D}$ deformable model segmentation using level set. The 2D method uses an external force field that is based on magnetostatics and hypothesized magnetic interactions between the active contour and object boundaries. The major contribution of the method is that the interaction of its forces can greatly improve the active contour in capturing complex geometries and dealing with difficult initializations, weak edges and broken boundaries. This method is then generalized to 3D by reformulating its external force based on geometrical interactions between the relative geometries of the deformable model and the object boundary characterized by image gradient. The evolution of the deformable model is solved using the level set method so that topological changes are handled automatically. The relative geometrical configurations between the deformable model and the object boundaries contribute to a dynamic vector force field that changes accordingly as the deformable model evolves. The geometrically induced dynamic interaction force has been shown to greatly improve the deformable model performance in acquiring complex geometries and highly concave boundaries, and it gives the deformable model a high invariancy in initialization configurations. The voxel interactions across the whole image domain provide a global view of the object boundary representation, giving the external force a long attraction range. The bidirectionality of the external force field allows the new deformable model to deal with arbitrary cross-boundary initializations, and facilitates the handling of weak edges and broken boundaries.
\end{abstract}

Xianghua Xie

Swansea University, Department of Computer Science, Faraday Tower, Singleton Park, Swansea SA2 8PP, United Kingdom, e-mail: x.xie@ swansea.ac.uk

Majid Mirmehdi

Bristol University, Department of Computer Science, Merchant Ventures Building, Bristol BS8 1UB, United Kingdom, e-mail: majidecs.bris.ac.uk

Si Yong Yeo, Igor Sazonov and Perumal Nithiarasu

Swansea University, College of Engineering, Talbot Building, Singleton Park, Swansea SA2 8PP, United Kingdom. 


\section{Introduction}

Depending on the assumption of how object boundary is described, active contours can be classified into edge based [Caselles et al(1997)Caselles, Kimmel, and Sapiro, Xu and Prince(1998), Paragios et al(2004)Paragios, Mellina-Gottardo, and Ramesh, Li et al(2005)Li, Liu, and Fox], region based [Chan and Vese(2001), Paragios and Deriche(2002), Cremers et al(2007)Cremers, Rousson, and Deriche], and hybrid approaches [Haddon and Boyce(1990), Chakraborty et al(1996)Chakraborty, Staib, and Duncan, Xie and Mirmehdi(2004)]. For edge based methods, it is assumed that object boundaries collocate with image intensity discontinuities which is widely adopted, for example, in depth estimation from stereo [Birchfield and Tomasi(1999)]. Region based techniques, on the other hand, assume that object boundaries collocate with discontinuities in regional characteristics, such as color and texture. In other words, each object has its own distinctive and continuous regional features.

Region based techniques have some obvious advantages over edge based methods in that object boundary description based on image gradient can often be compromised by noise and weak edges. They are also less sensitive to initialization, while edge based active contours are prone to local minima. Thus, it is often desirable for edge based techniques to carefully place the initial contour. This assumes that the prior knowledge of the object location is available, which is not always true in reality. Existing techniques can only reduce this initialization dependency to a very limited extent. The balloon force [Caselles et al(1997)Caselles, Kimmel, and Sapiro] can only expand or shrink the contours. The bidirectionality of GVF can sometimes cause the contours to collapse on approach to the same boundary. Moreover, it has convergence issues caused by critical points. [Gil and Radeva(2003), Paragios et al(2004)Paragios, Mellina-Gottardo, and Ramesh, Xie and Mirmehdi(2008)]. It is evidently clear that initialization invariance is particularly difficult to achieve for edge based methods. More recent attempts, such as [Gil and Radeva(2003), Paragios et al(2004)Paragios, Mellina-Gottardo, and Ramesh, Jalba et al(2004)Jalba, Wilkinson, and Roerdink, Li et al(2005)Li, Liu, and Fox], showed promising but limited success.

In this chapter, we present an image gradient based approach to perform $2 \mathrm{D}$ and 3D deformable model segmentation using level set. Section 2 presents the 2D method which uses an external force field that is based on magnetostatics and hypothesized magnetic interactions between the active contour and object boundaries. The major contribution of the method is that the interaction of its forces can greatly improve the active contour in capturing complex geometries and dealing with difficult initializations, weak edges and broken boundaries. This method is then generalized to 3D in Section 3 by reformulating its external force based on geometrical interactions between the relative geometries of the deformable model and the object boundary characterized by image gradient. The relative geometrical configurations between the deformable model and the object boundaries contribute to a dynamic vector force field that changes accordingly as the deformable model evolves. Experimental results are shown in Section 4. The proposed dynamic inter- 
action force has been shown to greatly improve the deformable model performance in acquiring complex geometries and highly concave boundaries, and it gives the deformable model a high invariancy in initialization configurations. The voxel interactions across the whole image domain provide a global view of the object boundary representation, giving the external force a long attraction range. The bidirectionality of the external force field allows the new deformable model to deal with arbitrary cross-boundary initializations, and facilitates the handling of weak edges and broken boundaries.

\section{MAC model: a 2D approach}

Fittings based on local intensity discontinuity can often lead to undesired local minima. The CPM [Jalba et al(2004)Jalba, Wilkinson, and Roerdink] assigns opposite charges to edges and free particles so that the particles are pulled towards edges while repelling each other. This global interaction provides much freedom of initialization. However, particles on weak edge can be gradually pulled towards neighboring strong edges, resulting in broken boundaries. Particle addition and deletion and contour reconstruction can also be difficult in practice.

Instead of assigning fixed charges, we allow the charges flow through the edges. These flows of charges will then generate a magnetic field. The active contour, carrying similar flow of charges, will be attracted towards the edges under this magnetic influence. Without losing generality, let us consider the image plane as a 2D plane in a $3 \mathrm{D}$ space whose origin coincides with the origin of the image coordinates. Additionally, the third dimension of this $3 \mathrm{D}$ space is considered perpendicular to the image plane.

The direction of the currents, flows of charges, running through object boundary can be estimated based on edge orientation, which can be conveniently obtained by a $90^{\circ}$ rotation in the image plane of the normalized gradient vectors $\left(\hat{I}_{x}, \hat{I}_{y}\right)$, where $I$ denotes an image. Let $\mathbf{x}$ denote a point in the image domain. Thus, the object boundary current direction, $\mathbf{O}(\mathbf{x})$, can be estimated as: $\mathbf{O}(\mathbf{x})=(-1)^{\lambda}\left(-\hat{I}_{y}(\mathbf{x}), \hat{I}_{x}(\mathbf{x}), 0\right)$, where $\lambda=1$ gives an anti-clockwise rotation in the image coordinates, and $\lambda=2$ provides a clockwise rotation. However, we show later by using the proposed level set updating scheme different $\lambda$ values lead to the same result. Since the active contour is embedded in a signed distance function, the direction of current for the contour, denoted as $v$, can be similarly obtained by rotating the gradient vector $\nabla \Phi$ of the level set function. Similar to $\mathbf{O}, v$ is also three dimensional and lies in the image domain, i.e. $v(\mathbf{x})=\left(-\hat{\Phi}_{y}(\mathbf{x}), \hat{\Phi}_{x}(\mathbf{x}), 0\right)$.

Let $f(\mathbf{x})$ be the magnitude of edge pixel and the magnitude of boundary current be proportional to edge strength, that is, the electric current on object boundary is defined as $f(\mathbf{x}) \mathbf{O}(\mathbf{x})$. The magnetic flux $\mathbf{B}(\mathbf{x})$ generated by gradient vectors at each pixel position $\mathbf{x}$ can then be computed as: 


$$
\mathbf{B}(\mathbf{x}) \propto \sum_{\mathbf{s} \in \mathbf{S}, \mathbf{s} \neq \mathbf{x}} f(\mathbf{s}) \mathbf{O}(\mathbf{s}) \times \frac{\hat{\mathbf{R}}_{\mathbf{x s}}}{R_{\mathbf{x s}}^{2}},
$$

where $\mathbf{s}$ denotes an edge pixel position, $\mathbf{S}$ is the set containing all the edge pixel positions across the image, $\hat{\mathbf{R}}_{\mathbf{x s}}$ denotes a $3 \mathrm{D}$ unit vector from $\mathbf{x}$ to $\mathbf{s}$ in the image plane, and $R_{\mathbf{x s}}$ is the distance between them. Thresholding can be applied to remove some erroneous edge pixels with very small gradient magnitude [Jalba et al(2004)Jalba, Wilkinson, and Roerdink, Xie and Mirmehdi(2008)]. The active contour is assigned with unit magnitude of electric current. The force imposed on it can be derived as:

$$
\mathbf{F}(\mathbf{x}) \propto v(\mathbf{x}) \times \mathbf{B}(\mathbf{x}) .
$$

From (1) and (2), we can see that $\mathbf{B}$ intersects the image plane perpendicularly and $\mathbf{F}$ is always perpendicular to both $v$ and $\mathbf{B}$. Thus, $\mathbf{F}$ also lies in the image domain and its third element equals to zero. For simplicity, from now on, we shall ignore its third dimensional component and denote $\mathbf{F}(\mathbf{x})$ as a $2 \mathrm{D}$ vector field in the image domain. The basic model can then be formulated as:

$$
\frac{\mathrm{d} C}{\mathrm{~d} t}=\alpha g(\mathbf{x}) \kappa \hat{\mathbf{n}}+(1-\alpha)(\mathbf{F}(\mathbf{x}) \cdot \hat{\mathbf{n}}) \hat{\mathbf{n}},
$$

where $g(\mathbf{x})=1 /(1+f(\mathbf{x})), \kappa$ denotes the curvature, and $\hat{\mathbf{n}}$ is inward unit normal. Its level set representation then takes this form:

$$
\frac{\partial \Phi}{\partial t}=\alpha g(\mathbf{x}) \nabla \cdot\left(\frac{\nabla \Phi}{|\nabla \Phi|}\right)|\nabla \Phi|-(1-\alpha) \mathbf{F}(\mathbf{x}) \cdot \nabla \Phi
$$

We can see from (1) and (2) that the image force is derived from global interactions among rotated gradient vectors, i.e. $f(\mathbf{x}) \mathbf{O}(\mathbf{x})$. Thus, it is more robust than fittings based on local gradient towards weak edges (where $f(\mathbf{x})$ is small) and noise (where $\mathbf{O}(\mathbf{x})$ is locally inconsistent). It is worth noting, however, that general contrast consistency along the object boundaries is important to the model. Large contrast variation can disrupt the force field, e.g. half of the object appears brighter than background and the other half appears to be darker. However, this does not mean that the entire object has to be brighter or darker than background. Those regions away from object boundary can be continuously varying in intensity. The model also can tolerate a fair amount of local contrast inconsistency, in the same way as to image noise and weak/broken edges.

As aforementioned, due to cross product computation the external force, $\mathbf{F}$, is always perpendicular to $v$ which is tangent to the contour, i.e. the external force is imposed along the normal direction. Note the internal force due to curvature flow is enforced in the inward normal direction. Thus, the total force is always perpendicular to active contour. In other words, it dynamically updates itself according to contour evolution to push and pull the contours along the normal direction until they reach object boundaries where forces from both sides are in balance. As a result, the propagating contour will not suffer from those convergence issues related to 
static force fields, such as GVF, in which evolving contours may become tangent to underlying force vectors resulting in false convergence. This force field is also significantly different from others used in edge based methods. For example, in CPM, the force between an edge pixel $\mathbf{s}$ and an infinitesimal contour segment $\mathbf{c}$ lies in a straight line between these two, regardless the orientation of the contour segment. In our model, the orientation of the edge pixel and the contour segment also have influence on the resulting force interaction. This ability to adapt is very important since it ensures the active contour, once initialized, overcome deep concavities and narrow regions to reach object boundaries.

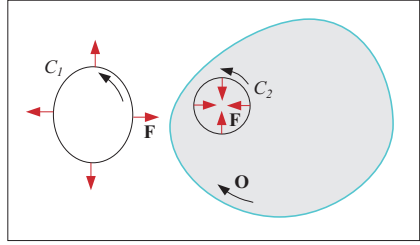

(A)

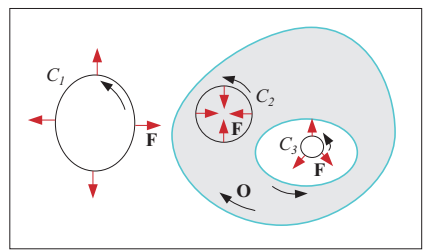

(C)

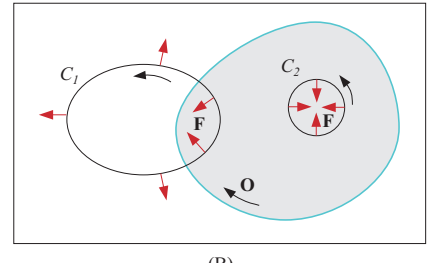

(B)

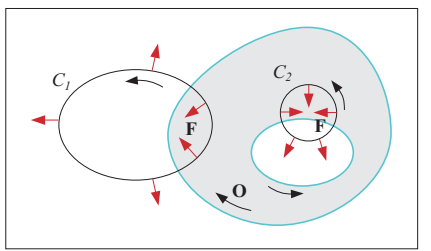

(D)

Fig. 1 Preventing contour collapsing. (a): Two contours, $C_{1}$ and $C_{2}$, are placed on each side of an object boundary with current directions indicated by arrows. Contour $C_{1}$ is attracted by the object boundary and expands itself in the outward normal direction. It eventually will wrap around and capture the object boundary. Contour $C_{2}$ however is repelled and forced to shrink in the inward normal direction. Thus, two contours will not collapse to each other. (b): Similar to (a), however contour $C_{1}$ is placed across the object boundary. Those contour segments of $C_{1}$ that are inside object boundary will be pulled towards object boundary and the rest of contour $C_{1}$ will expand and wrap around the object boundary. The segments inside object boundary and outside will not collapse to each other. (c): The object in this case contains an internal boundary. The behavior of $C_{1}$ and $C_{2}$ is similar to that in (a). Contour $C_{3}$ will expand itself to capture the internal boundary. Three contours will not collapse to each other, while capturing both boundaries. (d): Contours $C_{1}$ and $C_{2}$ are now initialized across external and internal boundaries, respectively. The behavior of $C_{1}$ is similar to that in (b). The contour segments of $C_{2}$ that are inside the object (gray area) will be attracted to the object internal boundary that is initially inside contour $C_{2}$. The other contour segments of $C_{2}$ will expand to capture the rest internal boundaries. No contour collapsing will occur, either. GVF contours, as an example, will collapse to each other in all above scenarios.

By incorporating (2), Eqn. (3) can be re-written as:

$$
\begin{aligned}
\frac{\mathrm{d} C}{\mathrm{~d} t} & =\alpha g(\mathbf{x}) \kappa \hat{\mathbf{n}}+(1-\alpha)(v(\mathbf{x}) \times \mathbf{B}(\mathbf{x}) \cdot(\hat{\mathbf{n}}, 0)) \hat{\mathbf{n}} \\
& =\alpha g(\mathbf{x}) \kappa \hat{\mathbf{n}}+(1-\alpha)(\mathbf{B}(\mathbf{x}) \cdot((\hat{\mathbf{n}}, 0) \times v(\mathbf{x}))) \hat{\mathbf{n}} .
\end{aligned}
$$


The external force in the second term is in fact a projection of the magnetic flux onto a binormal unit vector which is computed from a cross product of the contour inward normal and its tangent vector. A positive projection will force the contour to expand and a negative projection will shrink the contour, which acts in a similar way as what a region indication function does in a region based approach, however, this is derived from the edge based assumption. Thus, an edge can attract or push a contour which may lie either side of the edge. However, this bidirectionality is fundamentally different from that in, for example, GVF. In GVF, the force imposed on the contour is independent of the contour itself, which can cause the contours to collapse to each other when reaching to the same object boundary. For the proposed method, the force is related to both the image gradient and the contour (which can be clearly seen from Eqn. (5)). It has the ability to prevent the contour from reaching to the same boundary and disappearing after merging together.

In [Xie and Mirmehdi(2008)], we proposed to perform nonlinear diffusion of the magnetic field in order to overcome noise interference when necessary. An edge saliency measure can be added to the weighting function in order to better preserve the edges [Xie(2010)]. Let $B(\mathbf{x})$ denote the signed magnitude of $\mathbf{B}(\mathbf{x})$. The diffused field $\hat{\mathscr{B}}(\mathbf{x})$ is obtained by solving:

$$
\frac{\mathrm{d} \mathscr{B}}{d t}(\mathbf{x})=p(B(\mathbf{x})) \nabla^{2} \mathscr{B}(\mathbf{x})-q(B(\mathbf{x}))(\mathscr{B}(\mathbf{x})-B(\mathbf{x}))
$$

where $p(B(\mathbf{x}))=e^{-\frac{|B(\mathbf{x})| \mathscr{S}(\mathbf{x})}{K}}, q()=.1-p($.$) , and \mathscr{S}($.$) is an edge saliency measure$ which is measured based on edge strength and orientation coherency, i.e. $\mathscr{S}(\mathbf{x})=$ $f(\mathbf{x}) v(\mathbf{x})$ where $v($.$) is the variance of orientation in a local neighborhood, e.g. 9 \times 9$ as used here. More sophisticated saliency measures, e.g. [Heidemann(2005)], can be used. Weighting the flux magnitude with $\mathscr{S}($.$) further ensures as little diffusion$ as possible at object boundaries, while areas lack of consistent support from edges result in substantial diffusion.

\section{Extenstion to 3D}

Shape segmentation from volumetric data has an important role in applications such as medical image analysis. Volumetric image segmentation remains an intricate process, due to the complexity and variability of image data and shapes (i.e. anatomical structures). There have been applications of simple techniques such as thresholding and region growing in the extraction of 3D objects from volumetric images [Smith et al(2007)Smith, Smith, Williams, Rodriguez, and Hoying, Wu et al(2008)Wu, Ye, $\mathrm{Ma}$, Sun, $\mathrm{Xu}$, and Cui]. However, these techniques are very sensitive to noise and intensity inhomogeneities which exist in real images, and often produce leakages and regions which are not contiguous. Statistical approaches [Ruan et al(2000)Ruan, Jaggi, Xue, Fadili, and Bloyet, Hao and Li(2007)] are also used to identify different tissue structures from medical images. It usually involves manual interaction 
to segment images in order to obtain a sufficiently large set of training samples. Such strategies are often restricted to problems where there is sufficient prior knowledge about the shape or appearance variations of the relevant structures. Also, the use of the same training set for a large number of image scans may lead to biased results that do not take sufficient consideration of the variability within individuals. Atlas based approaches perform segmentation based on image registration techniques [Maintz and Viergever(1998)], whereby an image can be segmented by finding a transformation that maps a template image to the target image. It is however generally difficult for atlas based techniques to accurately extract complex geometries such as those from volumetric medical images due to the variability of anatomical structures.

The external force field presented previously is based on the hypothesized magnetic force between the active contour and object boundaries. This formulation can be applied directly in the magnetostatic active contour to compute the magnetic field and force required to draw the active contour towards object boundaries in 2D images. This image gradient based method showed significant improvements on convergence issues, e.g. reaching deep concavities, and in handling weak edges and broken boundaries. When applying the analogy directly to deformable modeling, it requires estimation of tangent vectors for the deformable contours, which is convenient in $2 \mathrm{D}$ case, however, not possible in $3 \mathrm{D}$. Our approach is to define a novel external force field that is based on hypothesized geometrically induced interactions between the relative geometries of the deformable model and the object boundaries (characterized by image gradients). In other words, the magnitude and direction of the interaction forces are based on the relative position and orientation between the geometries of the deformable model and image object boundaries, and hence, it is called the geometric potential force (GPF) field [Yeo et al(2011)Yeo, Xie, Sazonov, and Nithiarasu]. The bidirectionality of the new external force field can facilitate arbitrary cross-boundary initialization, which is a very useful feature to have, especially in the segmentation of complex geometries in 3D. It also improves the performance of the deformable model in handling weak edges. In addition, the proposed external force field is dynamic in nature as it changes according to the relative position and orientation between the evolving deformable model and object boundary. This GPF force however is in fact a 3D extension of the 2D MAC model.
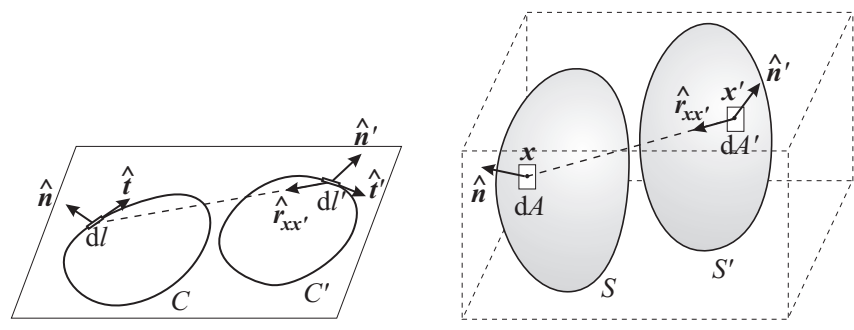

Fig. 2 Relative position and orientation between geometries in 2D and 3D. 


\section{1 geometric potential force}

First, consider a deformable contour $C$ and an ideal object boundary $C^{\prime}$ in the image plane (see Fig. 2). Let $\mathrm{d} l$ and $\mathrm{d} l^{\prime}$ denote the infinitesimal elements of contour $C$ and object boundary $C^{\prime}$, respectively. In the existing force field based models such as [Yang et al(2006)Yang, Mirmehdi, and Xie, Li et al(2007)Li, Kao, Gore, and Ding], the interaction between $\mathrm{d} l$ and $\mathrm{d} l^{\prime}$ is inversely proportional to the distance separating these two elements and the derived force lies in a straight line between them. They do not take into account the local geometry of the deformable contour $C$ or object boundary $C^{\prime}$. We propose to incorporate the mutual location and orientation of these elements.

Let $\mathbf{x}$ and $\mathbf{x}^{\prime}$ denote the positions of elements $\mathrm{d} l$ and $\mathrm{d} l^{\prime}$, respectively. Thus, $\mathbf{r}_{\mathbf{x x}}=$ $\mathbf{x}-\mathbf{x}^{\prime}$ is their mutual location of those two elements, $r_{\mathbf{x x}^{\prime}}=\left|\mathbf{x}-\mathbf{x}^{\prime}\right|$ is the distance between them, and $\hat{\mathbf{r}}_{\mathbf{x x}}=\left(\mathbf{x}-\mathbf{x}^{\prime}\right) / r_{\mathbf{x} \mathbf{x}^{\prime}}$ is the unit vector pointing $\mathrm{d} l$ from $\mathrm{d} l^{\prime}$. The directions of these elements can be represented by their unit tangent vectors $\hat{\mathbf{t}}$ and $\hat{\mathbf{t}}^{\prime}$. However, a unique tangent vector is no longer available for infinitesimal surface elements in 3D. Thus, we use unit outward normal vectors $\hat{\mathbf{n}}$ and $\hat{\mathbf{n}}^{\prime}$ to characterize the orientations of these elements instead (see Fig. 2). In 2D, they are simply $90^{\circ}$ rotated tangent vectors.

We are now ready to introduce the hypothesized interaction force $\mathrm{d} \mathbf{F} \mathrm{d} l$ which acts on element $\mathrm{d} l$ by virtue of the hypothesized force field induced by element $\mathrm{d} l^{\prime}$. It is desirable to combine the element orientation vectors and distance vector in deriving the force. We propose a simple but effective combination of these three vectors as $\hat{\mathbf{n}}\left(\hat{\mathbf{r}}_{\mathbf{x x}} \cdot \hat{\mathbf{n}}^{\prime}\right)$, unlike CPM [Jalba et al(2004)Jalba, Wilkinson, and Roerdink] as an example where only the distance vector $\hat{\mathbf{r}}_{\mathbf{x x}}$ is used. The multiplication of contour normal $\hat{\mathbf{n}}$ ensures that the force is always imposed in the normal direction so that the deformable model does not suffer from convergence issues (i.e. stationary points, saddle points and extreme boundary concavities), which are often associated with other vector force field based methods such as GVF [Xu and Prince(1998)]. The dot product of the object boundary element normal with the distance vector allows the force on the contour in the normal direction to diminish as the contour reaches the object boundary. Similar to other physics-inspired force field, it is also desirable to decay the force interaction with the increase of distance between the elements, i.e. the force is designed proportional to $\hat{\mathbf{n}}\left(\hat{\mathbf{r}}_{\mathbf{x x}^{\prime}} \cdot \hat{\mathbf{n}}^{\prime}\right) / r_{\mathbf{x x}^{\prime}}^{\lambda}$ where $\lambda>0$. Thus, the contribution of element $\mathrm{d} l^{\prime}$ of object boundary $C^{\prime}$ to the total force acting on $\mathrm{d} l$ in accordance with their distance and mutual orientation can be formulated as

$$
\mathrm{d} \mathbf{F} \mathrm{d} l=\hat{\mathbf{n}} \mathrm{d} G \mathrm{~d} l, \quad \mathrm{~d} G=\left(\frac{\hat{\mathbf{r}}_{\mathbf{x} \mathbf{x}^{\prime}}}{r_{\mathbf{x} \mathbf{x}^{\prime}}^{\lambda}} \cdot \hat{\mathbf{n}}^{\prime}\right) \mathrm{d} l^{\prime}
$$

where $\mathbf{F}$ is defined as force per unit length, $\mathrm{d} G$ is the contribution of element $\mathrm{d} l^{\prime}$ of object boundary $C^{\prime}$ into the scalar field $G(\mathbf{x})$, which can be considered as an intermediate potential field, and $\lambda$ is a positive constant that affects the magnitude of the interaction force based on the distance between the elements. In our study, we obtained the best results when $\lambda$ coincides with the dimension of the image 
data, i.e. $\lambda=2$ in the $2 \mathrm{D}$ case. Furthermore, we show later that when $\lambda$ coincides with data dimension in $2 \mathrm{D}$, the proposed force interaction has an explicit link to the magnetostatics theory and thus the spatial decay of the magnitude of the interaction force is analogous to that of the magnetic field.

As shown in (7), the computation of the new force field only requires unit normal vectors and relative position of the two elements, which is convenient to acquire. Thus, this new force field can be easily extended to higher dimensions, e.g. 3D. Let $\mathrm{d} A$ belong to the deformable surface $S$ whereas $\mathrm{d} A^{\prime}$ belongs to the object boundary $S^{\prime}$ (see Fig. 2). The generalized $3 \mathrm{D}$ version of force $\mathrm{d} \mathbf{F} \mathrm{d} A$ acting between these two area elements can be readily given as

$$
\mathrm{d} \mathbf{F} \mathrm{d} A=\hat{\mathbf{n}} \mathrm{d} G \mathrm{~d} A, \quad \mathrm{~d} G=\left(\frac{\hat{\mathbf{r}}_{\mathbf{x} \mathbf{x}^{\prime}}}{r_{\mathbf{x x}^{\prime}}^{\lambda}} \cdot \hat{\mathbf{n}}^{\prime}\right) \mathrm{d} A^{\prime}
$$

where $\mathbf{F}$ is defined as force per unit area, $G$ is the corresponding 3D potential field, $\hat{\mathbf{n}}$ and $\mathbf{n}^{\prime}$ are unit surface normals of the deformable model and object boundary, respectively, and $\lambda=3$. Again, the magnitude and direction of the induced force $\mathbf{F}$ is handled intrinsically by the relative position and orientation between the geometries of the deformable model determined by the evolving surface $S$ and object boundary determined by $S^{\prime}$. Since the force is derived geometrically and its interaction is a function of inverse distance, we name it geometric potential force (GPF).

\subsection{GPF Deformable Model}

The GPF force in (8) is derived using geometrical information from ideal object boundaries. Next, we extend this to deal with real image data and formulate it in 3D deformable modelling. In this work, we adopt an edge based approach, that is using image intensity discontinuity to estimate the presence and strength of object boundaries.

Let $I(\mathbf{x})$ denote the 3D image, where $\mathbf{x}$ is a voxel location in the image domain. Temporarily, we consider $\mathbf{x}$ as a continuously varying point. One may treat this as an interpolation between voxel grid points to obtain a continuous image $I(\mathbf{x})$. To compute the force acting on $\mathrm{d} A$, we first compute the total potential field for an arbitrary point $\mathbf{x}$ :

$$
G(\mathbf{x})=P . V . \oiiint_{S^{\prime}} \mathscr{W}\left(\mathbf{x}^{\prime}\right)\left(\frac{\hat{\mathbf{r}}_{\mathbf{x} \mathbf{x}^{\prime}}}{r_{\mathbf{x} \mathbf{x}^{\prime}}^{\lambda}} \cdot \hat{\mathbf{n}}^{\prime}\left(\mathbf{x}^{\prime}\right)\right) \mathrm{d} A^{\prime} .
$$

where $\mathscr{W}(\cdot)$ is a weighting function that is defined later, and P.V. means 'Principal Value': the contribution of infinitesimal circular vicinity of singular point $\mathbf{x}^{\prime}=\mathbf{x}$ into the integral is disregarded, which occurs when surfaces $S$ and $S^{\prime}$ intersect.

First, we consider the case, in which $S^{\prime}$ can be defined rigorously on an ideal object $O$, i.e. $S^{\prime}=\partial O$. The object $O$ can be specified by a binary image: 


$$
\mathscr{I}(\mathbf{x})=\left\{\begin{array}{cc}
\mathscr{I}_{0} & \mathbf{x} \in O \\
0 & \mathbf{x} \notin O
\end{array}\right.
$$

where $\mathscr{I}_{0}$ is a nonzero constant. For such an image, $\nabla \mathscr{I}$ is infinite on $S^{\prime}$ and can be represented through the 3D Dirac's delta as

$$
\nabla \mathscr{I}(\mathbf{x})=\Delta \mathscr{I} \delta\left(\mathbf{x}-\mathbf{x}^{\prime}\right) \hat{\mathbf{n}}^{\prime}\left(\mathbf{x}^{\prime}\right)
$$

where $\Delta \mathscr{I}$ is the jump in function $\mathscr{I}(\mathbf{x})$ at the boundary of $O ; \mathbf{x}^{\prime} \in S^{\prime}$ and $\hat{\mathbf{n}}^{\prime}\left(\mathbf{x}^{\prime}\right)$ is the unit normal vector to the surface $S^{\prime}$. Setting $\mathscr{W}$ equal to the jump of $\mathscr{I}$ at the boundary, i.e. $\mathscr{W}=\Delta \mathscr{I}$, we can re-write (9) as a volume integral

$$
G(\mathbf{x})=P . V . \iiint_{\Omega} \mathscr{W}\left(\mathbf{x}^{\prime}\right)\left(\frac{\hat{\mathbf{r}}_{\mathbf{x} \mathbf{x}^{\prime \prime}}}{r_{\mathbf{x} \mathbf{x}^{\prime \prime}}^{\lambda}} \cdot \hat{\mathbf{n}}^{\prime}\left(\mathbf{x}^{\prime}\right)\right) \delta\left(\mathbf{x}^{\prime \prime}-\mathbf{x}^{\prime}\right) \mathrm{d} V^{\prime \prime}
$$

Here, $\mathbf{x}^{\prime \prime}$ is the integration variable and $d V^{\prime \prime}$ denotes a volume element. The Dirac's delta is used to obtain the area element from the volume element, i.e. $d A^{\prime} \rightarrow \delta\left(\mathbf{x}^{\prime \prime}-\right.$ $\left.\mathbf{x}^{\prime}\right) d V^{\prime \prime}$.

Taking into account (11) and $\mathscr{W}=\Delta \mathscr{I}$, we can replace the product $\mathscr{W}\left(\mathbf{x}^{\prime}\right) \hat{\mathbf{n}}^{\prime}\left(\mathbf{x}^{\prime}\right) \delta\left(\mathbf{x}^{\prime \prime}-\right.$ $\left.\mathbf{x}^{\prime}\right)$ in the integral of (12) by $\nabla \mathscr{I}\left(\mathbf{x}^{\prime \prime}\right)$. Thus, (12) can be re-formulated as

$$
G(\mathbf{x})=P . V . \iiint_{\Omega}\left(\frac{\hat{\mathbf{r}}_{\mathbf{x x}} \mathbf{x}^{\prime \prime}}{r_{\mathbf{x x}^{\prime \prime}}^{\lambda}} \cdot \nabla \mathscr{I}\left(\mathbf{x}^{\prime \prime}\right)\right) \mathrm{d} V^{\prime \prime} .
$$

It is now readily generalizable to real $3 \mathrm{D}$ data.

In real images, $\nabla I$ is a smooth function reaching maximum magnitude in the vicinity of the object boundary. The natural generalization of (13) is to substitute Dirac's delta by this smoothed function analog into (13), i.e. $\mathscr{W}\left(\mathbf{x}^{\prime}\right) \hat{\mathbf{n}}^{\prime}\left(\mathbf{x}^{\prime}\right) \boldsymbol{\delta}\left(\mathbf{x}^{\prime \prime}-\right.$ $\left.\mathbf{x}^{\prime}\right) \rightarrow \nabla I\left(\mathbf{x}^{\prime \prime}\right)$, where $I$ denotes a real image. The geometric potential field in a continuous form can then be formulated as

$$
G(\mathbf{x})=P . V . \iiint_{\Omega}\left(\frac{\hat{\mathbf{r}}_{\mathbf{x} \mathbf{x}^{\prime}}}{r_{\mathbf{x} \mathbf{x}^{\prime}}^{\lambda}} \cdot \nabla I\left(\mathbf{x}^{\prime}\right)\right) \mathrm{d} V^{\prime} .
$$

Note, due to the substitution of $\mathscr{W}\left(\mathbf{x}^{\prime}\right) \hat{\mathbf{n}}^{\prime}\left(\mathbf{x}^{\prime}\right) \delta\left(\mathbf{x}^{\prime \prime}-\mathbf{x}^{\prime}\right)$ by $\nabla I\left(\mathbf{x}^{\prime \prime}\right)$, the $\mathbf{x}^{\prime}$ defined on the ideal surface $S^{\prime}$ is no longer needed. Hence, the notation is simplified by replacing the integral variable $\mathbf{x}^{\prime \prime}$ with $\mathbf{x}^{\prime}$. Finally, its discrete form can be written as

$$
G(\mathbf{x})=\sum_{\mathbf{x}^{\prime} \in \Omega, \mathbf{x}^{\prime} \neq \mathbf{x}}\left(\frac{\hat{\mathbf{r}}_{\mathbf{x} \mathbf{x}^{\prime}}}{r_{\mathbf{x} \mathbf{x}^{\prime}}^{\lambda}} \cdot \nabla I\left(\mathbf{x}^{\prime}\right)\right) .
$$

This can be considered as a convolution of the image gradient with the vector kernel $\mathbf{K}_{\lambda}(\mathbf{x})$ 


$$
\left\{\begin{array}{l}
\mathbf{K}_{\lambda}(\mathbf{x})=P . V \cdot \frac{\hat{\mathbf{x}}}{|\mathbf{x}|^{\lambda}}=P . V \cdot \frac{\mathbf{x}}{|\mathbf{x}|^{\lambda+1}} \\
G=\mathbf{K}_{\lambda} * \nabla I=\iiint_{\Omega}\left(\mathbf{K}_{\lambda}\left(\mathbf{x}-\mathbf{x}^{\prime}\right) \cdot \nabla I\left(\mathbf{x}^{\prime}\right)\right) d V^{\prime}
\end{array}\right.
$$

which can be computed efficiently using the fast Fourier transform (FFT). Note that the potential field $G$ is computed as a convolution of two vector functions.

The total force acting on the unit area element of the deformable surface $S$ is thus given as $\mathbf{F}=\hat{\mathbf{n}} G(\mathbf{x})$. where $\hat{\mathbf{n}}$ is the outward unit normal of level set surface. Note, an inward normal can also be used, i.e. $\mathbf{F}=-\hat{\mathbf{n}} G(\mathbf{x})$, which will result in opposite deformable model propagation since the force field is exactly in the opposite direction. Hence, the force can be re-written in a generalized form:

$$
\mathbf{F}=\mathscr{J} \hat{\mathbf{n}} G(\mathbf{x}) .
$$

where $\mathscr{J}$ is a constant taking values of \pm 1 . Note this is different from the constant force in the geodesic model, where the force is monotonically expanding or shrinking. The sign convention \pm is merely used to determine whether outward and inward normals of the deformable surface are considered.

The general contrast consistency along the object boundaries however is important to the model. Large contrast variation can disrupt the force field, e.g. half of the object appears brighter than background and the other half appears to be darker. However, this does not mean that the entire object has to be brighter or darker than background. Those regions away from object boundary can be continuously varying in intensity.

Once the force field $\mathbf{F}(\mathbf{x})$ is derived from the hypothesized interactions based on the relative geometries of the deformable model and object boundary is determined, the evolution of the deformable model $S(\mathbf{x}, t)$ under this GPF field can be given as

$$
\frac{\mathrm{d} S}{\mathrm{~d} t}=(\mathbf{F} \cdot \hat{\mathbf{n}}) \hat{\mathbf{n}} .
$$

Since surface smoothing is usually desirable, the mean curvature flow can be incorporated and the complete GPF deformable model evolution can be formulated as

$$
\frac{\mathrm{d} S}{\mathrm{~d} t}=\alpha g \kappa \hat{\mathbf{n}}+(1-\alpha)(\mathbf{F} \cdot \hat{\mathbf{n}}) \hat{\mathbf{n}}
$$

where $g(\mathbf{x})=1 /(1+|\nabla I|)$ is the edge stopping function. Note that in our case, the flow of $\mathbf{F}$ is directed by definition normal to surface $S$, therefore $(\mathbf{F} \cdot \hat{\mathbf{n}}) \hat{\mathbf{n}}=\mathbf{F}$. Notation $(\mathbf{F} \cdot \hat{\mathbf{n}}) \hat{\mathbf{n}}$ is inherited from the traditional methods, e.g. GGVF. The level set representation of the proposed deformable model based on GPF can then be written as

$$
\frac{\partial \Phi}{\partial t}=\alpha g \kappa|\nabla \Phi|-(1-\alpha)(\mathbf{F} \cdot \nabla \Phi)
$$

where $\Phi(t, \mathbf{x})$ is the level set function, such that the deformable surface $S$ is defined as $\Phi(t, \mathbf{x})=0$. Note, the GPF force field is defined on the deformable surface, which 
is implicitly embedded in the level set function, i.e. the force field computed at the propagating front needs to be extended across the computational domain so that the full level set function can be continuously evolved. Although direct force extension method such as [Adalsteinsson and Sethian(1998)] can be used, we can conveniently compute the GPF forces for each level set so that this external force is extended to the entire level set function.

The GPF deformable model differs from conventional edge based models by utilizing edge voxel interactions across the whole image, thus providing a more global view of the object boundary. The magnitude of the potential field strength at each image location $\mathbf{x}$ is based on the relative position of $\mathbf{x}$ with all other voxels in the image. Therefore, voxels at homogeneous regions will also have a non-zero potential field strength. In this way, surfaces which are initialized far away from object boundaries can propagate towards the image edges and converge.

As shown in (8), the dot product $\hat{\mathbf{r}}_{\mathbf{x x}} \cdot \hat{\mathbf{n}}^{\prime}$ can be both positive and negative, depending on the relative configurations of the geometries between the deformable model and the image boundaries, thus giving a bidirectional vector force field. This useful bidirectionality facilitates arbitrary cross boundary initializations, as its force vectors point towards the object boundary from both ways. This also allows the model to stabilize the deformable surfaces at weak edges, thus preventing leakage.

The physics-based deformable models described in [Li et al(2007)Li, Kao, Gore, and Ding, Jalba et al(2004)Jalba, Wilkinson, and Roerdink, Yang et al(2006)Yang, Mirmehdi, and Xie, Park and Chung(2002), Zhu et al(2008)Zhu, Zhang, Zeng, and Wang] all use a kernel based function to compute the external force field with kernels being decreasing functions of distance from the origin. They are in effect equivalent to the external force derived in [ $\mathrm{Li}$ et al(2007)Li, Kao, Gore, and Ding] based on convolving a vector field with the edge map. For example, the external force in [Jalba et al(2004)Jalba, Wilkinson, and Roerdink] can be represented as a convolution with the same kernel $\mathbf{K}_{\lambda}$ (16) with $\lambda=2$ :

$$
\mathbf{F}_{a}(\mathbf{x})=\frac{q}{4 \pi \varepsilon}\left(\mathbf{K}_{\lambda} *|\nabla I|\right), \quad \mathbf{F}_{r}(\mathbf{x})=\frac{q^{2}}{4 \pi \varepsilon}\left(\mathbf{K}_{\lambda} * 1_{\Omega}\right)
$$

where $1_{\Omega}(\mathbf{x})$ is a function equal to 1 when $\mathbf{x} \in \Omega$ and 0 otherwise. The repelling force is largely imposed in the tangential direction, which has very limited effect on changing the shape or topology of the deformable model. Thus, it is not necessary in our model. In order to compare with the dominant attraction force $\mathbf{F}_{a}$, we combine (16) and (17) and rewrite the GPF force as

$$
\mathbf{F}_{\mathrm{GPF}}=\mathscr{J} \hat{\mathbf{n}}\left(\mathbf{K}_{\lambda} * \nabla I\right)
$$

It is clear that the GPF force is directed by the normal of the deformable model, i.e. it does not contain the tangential 'parasitic' component in contrast to the $\mathbf{F}_{a}$ force. Moreover, the proposed GPF takes into account edge orientations, as well as edge strength (the convolution in (21) is based on a convolution of a vector function on a scalar field; whereas in (22) it is carried out on a vector field). 


\section{Experimental results}

In this section, we present experimental results on both synthetic and real world image data. The comparative analysis is performed using several classical and stateof-the-art methods, which consists of image gradient based and region based methods. In particular, the geodesic model is included as a representative of conventional local edge fitting based method which is based on monotonically expanding or shrinking force. The various vector field based models, such as [Li et al(2007)Li, Kao, Gore, and Ding, Jalba et al(2004)Jalba, Wilkinson, and Roerdink, Park and Chung(2002), Zhu et al(2008)Zhu, Zhang, Zeng, and Wang], have very similar convergence and initialization dependence behavior to the GVF or GGVF, since their dominant external forces are static as discussed earlier.

Fig. 3 shows comparative results of 2D segmentation on synthetic data. Even though these images have clear (ideal) boundary and the active contour models are all using level set representation, convergence issues still arise. The solution becomes particularly challenging under certain initialization conditions. The first two rows in Fig. 3 show comparative recovered shapes for the DVF [Cohen and Cohen(1993)], geodesic, GGVF, GeoGGVF, CVF, and MAC models in columns (b) to $(\mathrm{g})$ respectively. When the initial contour was placed outside the four discs (first row), only the geodesic snake and MAC could accurately recover them. However, in a more arbitrary cross-boundary initialization case (second row), only MAC was successful. Next, we consider the recovery of an acute concavity as shown in the third and fourth rows in Fig. 3, again with different initialization conditions. For the DVF, GGVF, and GeoGGVF snakes, their stationary vector force fields exhibit stationary and saddle points, e.g. the saddle point at the entrance of the concave shape which prevents the snake converging to the object boundaries. Again, given an arbitrary cross-boundary initialization, the geodesic snake suffers severe problems and the constriction on the left side of the concave shape causes difficulties for the CVF active contour. MAC was the only active contour model that could successfully recover the shape in both initializations. When dealing with complex geometries, such as the swirl shape and the text "PAMI" shown in the last two rows in Fig. 3, MAC was the only model that managed to fully recover the shapes. The latter example further illustrates MAC's ability in dealing with multiple objects with complex topology.

Fig. 4 shows a brain MRI image and its comparative segmentation results. For the active contour models, the snake was initialized across the left and right hemispheres, while for the particle model a grid of charges was used. The static vector force based methods (DVF, GGVF, and GeoGGVF) failed to evolve through the tortuous structures and collapsed to nearby edges as shown in rows (a), (c), and (d). The geodesic snake, in row (b), stepped across the weak edges but also failed to localize the boundaries. The free charges of CPM initially reached most of the object boundaries, but later failed to stabilize at weaker edges resulting in incomplete boundary description (row (e)). The MAC contours succeeded in evolving through the narrow and twisted structures as shown in row (f). Multiple regions were captured simultaneously. 
(a)
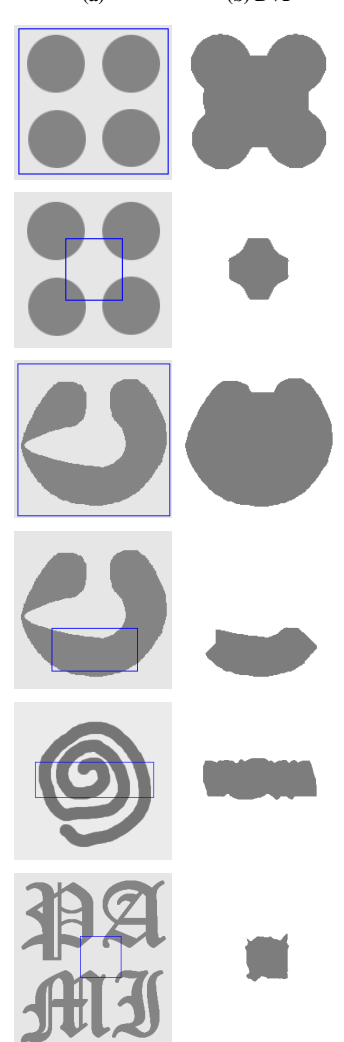

(c) Geodesic
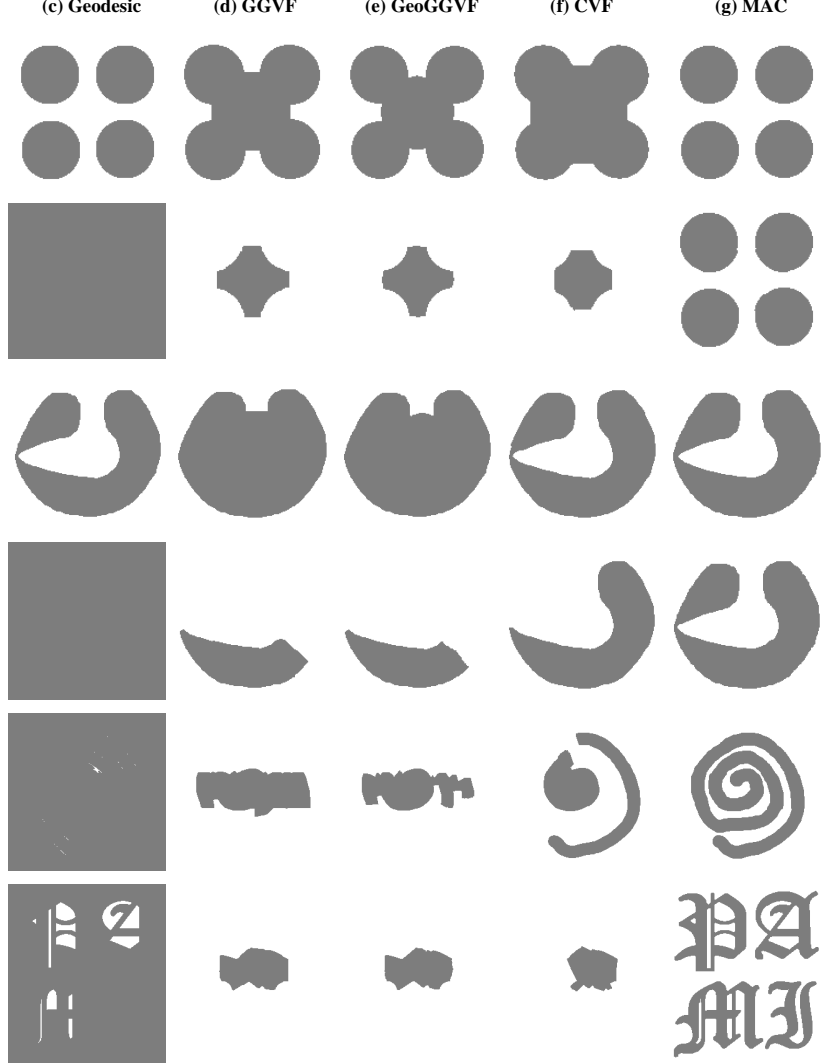
ered shape using DVF, (c) geodesic, (d) GGVF, (e) GeoGGVF, (f) CVF, and (g) proposed MAC snakes.

Next, we demonstrate the results of the 3D model. The first row of Fig. 5 shows a substantially blurred image with linearly varying intensity, and the corresponding edge map and computed geometric potential field. In addition, as the deformable model evolves, the unit vector $\hat{\mathbf{r}}_{\mathbf{x} \mathbf{x}^{\prime}}$ changes accordingly based on the relative geometries. This contributes to a vector force field that changes dynamically as the deformable model evolves, as depicted in the second row of Fig. 5. Therefore, the proposed model has much better invariance to its initial position and can deal with complex geometries and extreme boundary concavities.

Fig. 6 shows comparative results of extracting 3D shapes. The first column shows the shape extraction results for the six-ellipsoids problem. Given an arbitrary initialization across all the ellipsoids, only GPF could accurately recover the shapes. The geodesic model, given the same initialization configuration, simply expanded outwards and reached the image borders. This is due to the fact that the geodesic model cannot handle cross-boundary initialization as the constant pressure term can 

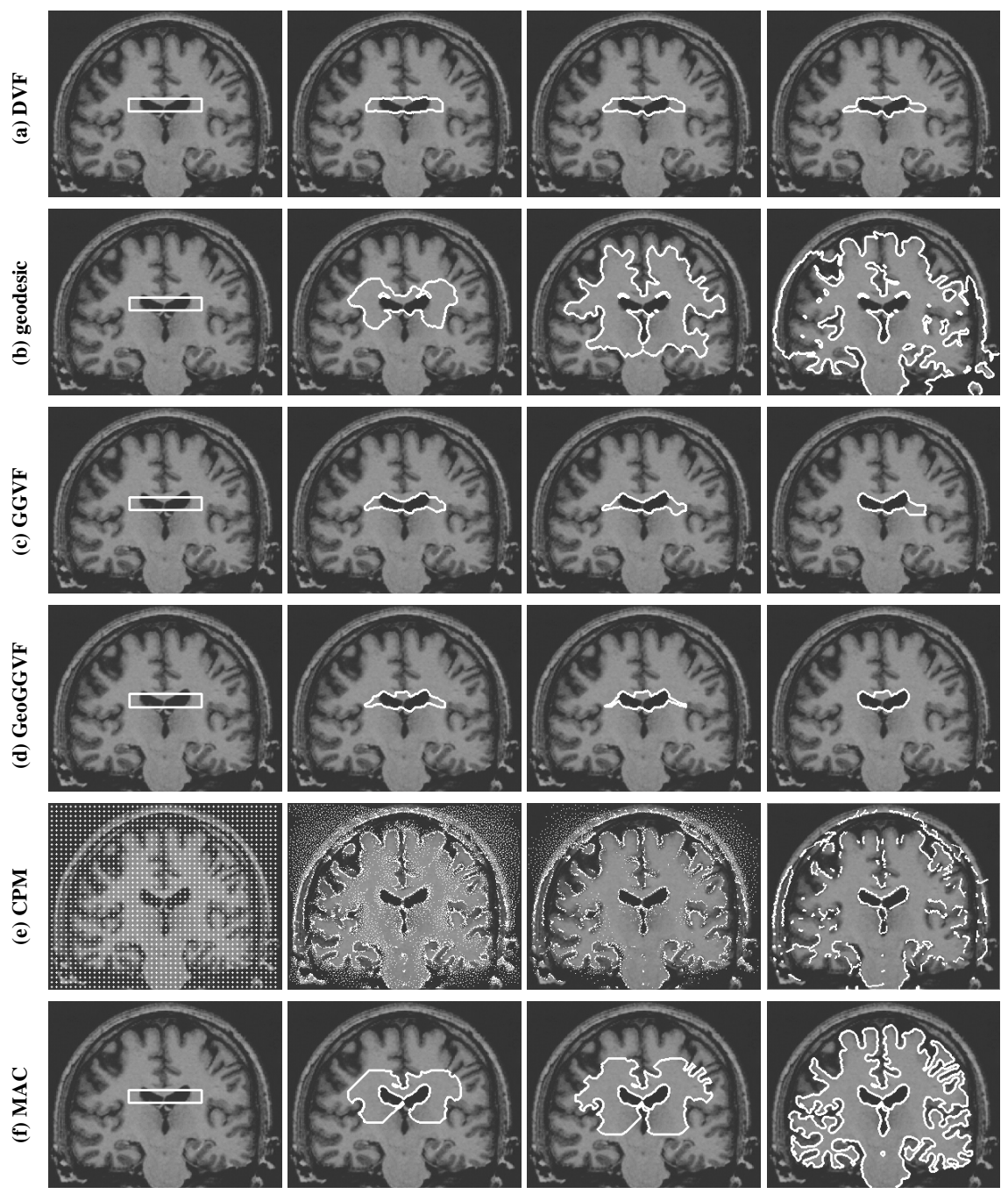

Fig. 4 Comparative study - results by row: (a) DVF, (b) geodesic, (c) GGVF, (d) GeoGGVF, (e) CPM, (f) MAC.

only monotonically shrink or expand the contour. Although the bidirectionality of the GGVF model enables it to handle cross-boundary initialization, the saddle and stationary points in this example prevented GGVF from extracting the ellipsoids. The second and third columns show the geometrical object to be recovered consists of two flattened ellipsoids connected by a narrowing tube with a constriction in the middle. With the deformable models initialized inside one of the ellipsoid, only GPF could propagate through the narrowing tube to accurately extract the shape. Also, with a more arbitrary cross-boundary initialization, GPF was the only suc- 

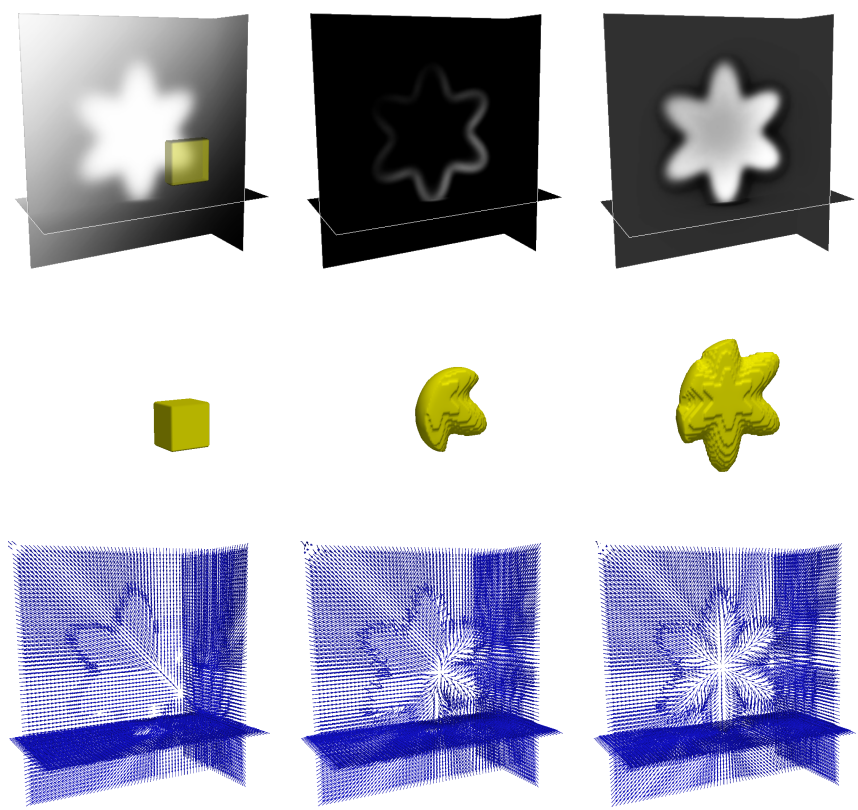

Fig. 5 GPF: first row from left to right - input image and initial deformable model, corresponding edge map and computed geometric potential field, second row - initial and evolving deformable models, and the third row - associated GPF vector field.

cessful model to extract the exact shape. The fourth and fifth columns in Fig. 6 compares the shape extraction results on a complex geometry with different initialization configurations. When the initial surface is placed inside one of the sphere of the molecular structure, GPF is the only model that managed to extract the geometry successfully. These examples demonstrate the superior performance of the GPF deformable model in resolving deep concavities and handling complex geometries and topologies. This is mainly due to the dynamic nature of the vector force field. In addition, we show that the bidirectionality of the new force field gives GPF the flexibility to deal with arbitrary cross-initializations.

Fig. 7 shows comparative results on the segmentation of cerebral arterial structure from magnetic resonance (MR) imaging. Two initial surfaces are placed inside the object of interest for the geodesic model, and across the object boundaries for GGVF, Chan-Vese and GPF. The geodesic model cannot propagate through the narrow tubular structures, and leaks out at weak object boundaries during the evolution. The GGVF model collapsed to the nearby object edges due to the saddle or stationary points inside the narrow image structures. In contrast, the Chan-Vese and GPF models are able to propagate through the long tubular structures to extract the cerebral arterial geometry. Further examples of the 3D method on real data are given in Fig. 8. The examples above have shown that the GPF deformable model can efficiently segment thin and complex structures, and can handle inhomogeneity in image intensities, noises and weak edges, which are often present in real images. 

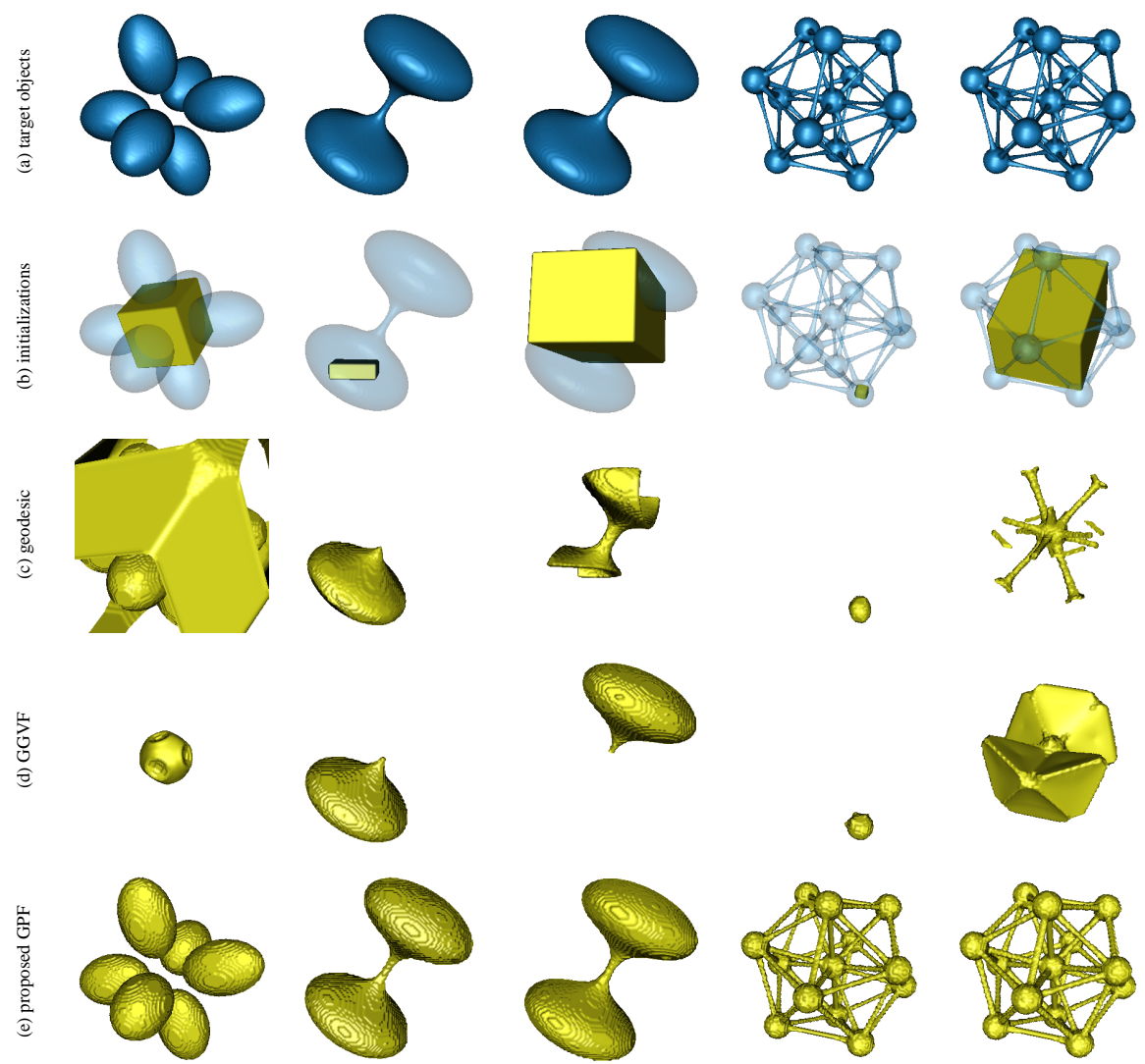

0

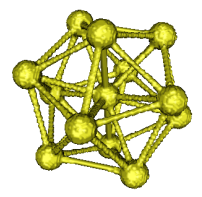

Fig. 6 Shape recovery from synthetic images: (a) isosurfaces of various shapes to be recovered from synthetic images $(128 \times 128 \times 128)$, (b) initial deformable models (yellow) with input shapes (blue, semi-transparent), (c) recovered shape using geodesic, (d) GGVF, (e) proposed GPF

The improvements achieved by the proposed method, as demonstrated extensively in various examples, are significant and consistent.

\section{Conclusions}

We have presented two image gradient based deformable models that are both based on global image gradient vector interations, instead of conventional local edge fitting. The 2D MAC model can attract the contour into deep concave regions and does not suffer from saddle point and stationary point problems. Our comparative study showed significant improvement in initialization invariancy and convergence capability on existing state-of-the-art techniques. Its extension to 3D, known as the geometric potential force $(\mathrm{GPF})$ model, utilizes pixel or voxel interactions across 

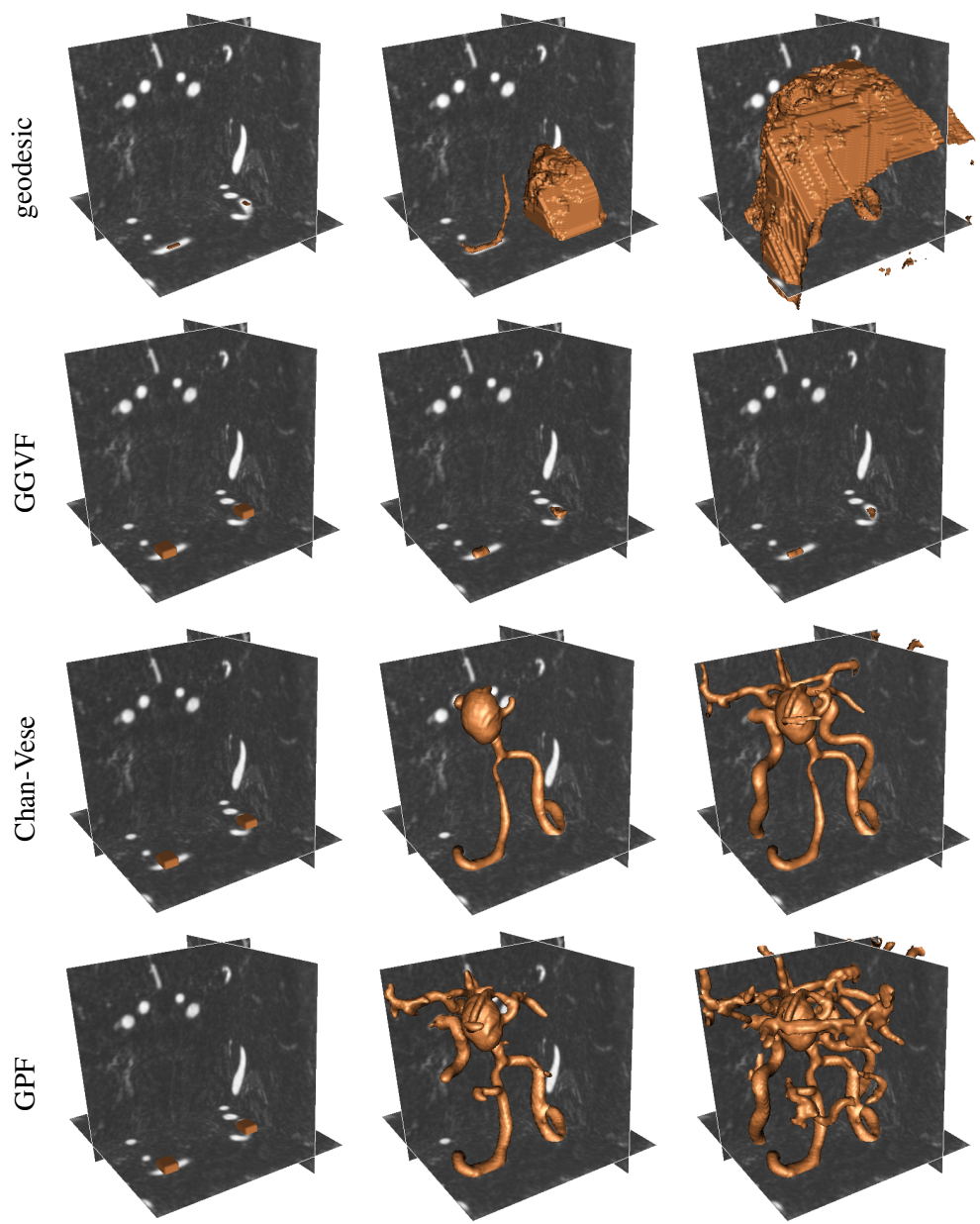

Fig. 7 Segmentation of cerebral arterial structure using different deformable models - first row: geodesic; second row: GGVF; third row: Chan-Vese; fourth row: proposed GPF.
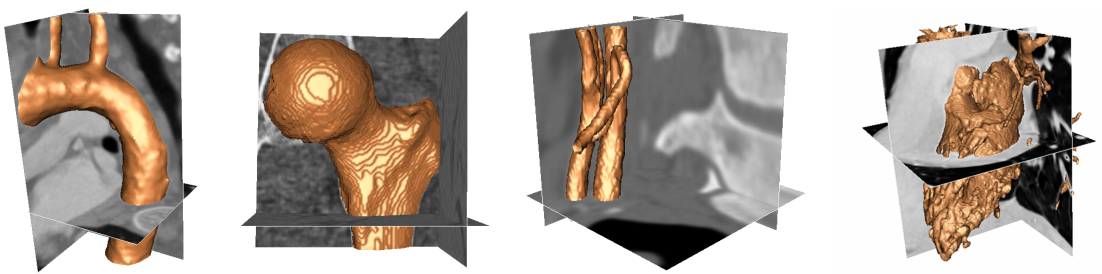

Fig. 8 More examples of the proposed method on real 3D medical data. 
the whole image. The derived geometric potential field is thus more informative and exhibits spatial and structural characteristics of image objects which are more coherent than image cues that are based solely on local edge or regional information. This makes the model more robust towards image noise and weak object edges. The relativity between geometries gives the proposed deformable model its distinctive bidirectionality, which facilitates the handling of arbitrary cross-boundary initializations. The straightforward generalization of the proposed model to higher dimensions allows the framework to be applied on $\mathrm{N}$-dimensional images, and opens up to a wide range of potential applications.

\section{References}

[Adalsteinsson and Sethian(1998)] Adalsteinsson D, Sethian J (1998) The fast construction of extension velocities in level set methods. J Comp Phy 148:2-22

[Birchfield and Tomasi(1999)] Birchfield S, Tomasi C (1999) Depth discontinuities by pixel-topixel stereo. IJCV 35(3):269-293

[Caselles et al(1997)Caselles, Kimmel, and Sapiro] Caselles V, Kimmel R, Sapiro G (1997) Geodesic active contour. IJCV 22(1):61-79

[Chakraborty et al(1996)Chakraborty, Staib, and Duncan] Chakraborty A, Staib H, Duncan J (1996) Deformable boundary finding in medical images by integrating gradient and region information. IEEE T-MI 15(6):859-870

[Chan and Vese(2001)] Chan T, Vese L (2001) Active contours without edges. IEEE T-IP 10(2):266-277

[Cohen and Cohen(1993)] Cohen L, Cohen I (1993) Finite-element methods for active contour models and balloons for 2-D and 3-D images. IEEE T-PAMI 15(11):1131-1147

[Cremers et al(2007)Cremers, Rousson, and Deriche] Cremers D, Rousson M, Deriche R (2007) A review of statistical approaches to level set segmentation: Integrating color, texture, motion and shape. IJCV 72(2):195-215

[Gil and Radeva(2003)] Gil D, Radeva P (2003) Curvature vector flow to assure convergent deformable models for shape modelling. In: EMMCVPR, pp 357-372

[Haddon and Boyce(1990)] Haddon J, Boyce J (1990) Image segmentation by unifying region and boundary information. IEEE T-PAMI 12:929-948

[Hao and Li(2007)] Hao J, Li M (2007) A supervised bayesian method for cerebrovascular segmentation. WSEAS Trans Signal Processing 3(12):487-495

[Heidemann(2005)] Heidemann G (2005) The long-range saliency of edge- and corner-based saliency points. IEEE T-IP 14:1701-1706

[Jalba et al(2004)Jalba, Wilkinson, and Roerdink] Jalba A, Wilkinson M, Roerdink J (2004) CPM: A deformable model for shape recovery and segmentation based on charged particles. IEEE T-PAMI 26(10):1320-1335

[Li et al(2005)Li, Liu, and Fox] Li C, Liu J, Fox M (2005) Segmentation of edge preserving gradient vector flow: an approach toward automatically initializing and splitting of snakes. In: CVPR, pp 162-167

[Li et al(2007)Li, Kao, Gore, and Ding] Li C, Kao C, Gore J, Ding Z (2007) Implicit active contours driven by local binary fitting energy. In: CVPR, pp 1-7

[Maintz and Viergever(1998)] Maintz JB, Viergever MA (1998) A survey of medical image registration. Medical Image Analysis 2(1):1-36

[Paragios and Deriche(2002)] Paragios N, Deriche R (2002) Geodesic active regions and level set methods for supervised texture segmentation. IJCV 46(3):223-247

[Paragios et al(2004)Paragios, Mellina-Gottardo, and Ramesh] Paragios N, Mellina-Gottardo O, Ramesh V (2004) Gradient vector flow geometric active contours. IEEE T-PAMI 26(3):402407 
[Park and Chung(2002)] Park HK, Chung MJ (2002) External force of snake: virtual electric field. Electronics Letters 38(24):1500-1502

[Ruan et al(2000)Ruan, Jaggi, Xue, Fadili, and Bloyet] Ruan S, Jaggi C, Xue J, Fadili J, Bloyet D (2000) Brain tissue classification of magnetic resonance images using partial volume modeling. IEEE T-MI 19(12):1179-1187

[Smith et al(2007)Smith, Smith, Williams, Rodriguez, and Hoying] Smith CM, Smith J, Williams SK, Rodriguez JJ, Hoying JB (2007) Automatic thresholding of three-dimensional microvascular structures from confocal microscopy images. J Microscopy 225(3):244-257

[Wu et al(2008)Wu, Ye, Ma, Sun, Xu, and Cui] Wu J, Ye F, Ma J, Sun X, Xu J, Cui Z (2008) The segmentation and visualization of human organs based on adaptive region growing method. In: Int. Conf. Comp. and Info. Tech., pp 439-443

[Xie(2010)] Xie X (2010) Active contouring based on gradient vector interaction and constrained level set diffusion. IEEE T-IP 19(1):154-164

[Xie and Mirmehdi(2004)] Xie X, Mirmehdi M (2004) RAGS: Region-aided geometric snake. IEEE T-IP 13(5):640-652

[Xie and Mirmehdi(2008)] Xie X, Mirmehdi M (2008) MAC: Magnetostatic active contour. IEEE T-PAMI 30(4):632-646

[Xu and Prince(1998)] Xu C, Prince J (1998) Snakes, shapes, \& gradient vector flow. IEEE T-IP $7(3): 359-369$

[Yang et al(2006)Yang, Mirmehdi, and Xie] Yang R, Mirmehdi M, Xie X (2006) A charged active contour based on electrostatics. In: ACIVS, pp 173-184

[Yeo et al(2011)Yeo, Xie, Sazonov, and Nithiarasu] Yeo S, Xie X, Sazonov I, Nithiarasu P (2011) Geometrically induced force interaction for three-dimensional deformable models. IEEE T-IP 20(5):1373-1387

[Zhu et al(2008)Zhu, Zhang, Zeng, and Wang] Zhu G, Zhang S, Zeng Q, Wang C (2008) Anisotropic virtual electric field for active contours. Pattern Recgnition Letters 29(11):16591666 